\title{
ANGPTL4 mediates the protective role of PPARy activators in the pathogenesis of preeclampsia
}

\author{
Lei Liu ${ }^{1,2}$, Xu Zhuang ${ }^{1}$, Meng Jiang ${ }^{1}$, Fei Guan ${ }^{1}$, Qin Fu' ${ }^{1}$ and Jianhua Lin ${ }^{\star, 1,2}$
}

Peroxisome proliferator-activated receptor $\gamma$ (PPAR $\gamma$ ) has been shown to be a therapeutic target for preeclampsia (PE). Angiopoietin-like protein 4 (ANGPTL4) is a multifunctional secretory protein involved in regulating lipid metabolism and angiogenesis in various tissues. However, the expression of PPAR $\gamma$ and ANGPTL4 and their interaction in PE remain elusive. Here we showed that PPAR $\gamma$ agonist rosiglitazone upregulated the expression and secretion of ANGPTL4 in a dose-dependent manner in HTR8/SVneo cells, human umbilical vein endothelial cells (HUVECs) and placental explants. More importantly, we confirmed that the PPAR $\gamma /$ retinoid X receptor $\alpha$ heterodimer specifically binds to the ANGPTL4 promoter region and enhances its transcriptional activity. In addition, the levels of ANGPTL4 and PPAR $\gamma$ activators in the serum and their expression in placental tissues were significantly reduced in preeclamptic patients compared with normal pregnant subjects. Furthermore, functional studies demonstrated that ANGPTL4 mediates the facilitative effects of the PPAR $\gamma$ agonist on the survival, proliferation, migration and invasion of HTR8/SVneo cells, placental explants outgrowth and angiogenesis in HUVECs. Taken together, our results suggest that ANGPTL4 is a potential target gene for PPAR $\gamma$ and mediates the protective role of PPAR $\gamma$ activators in the pathogenesis of PE. Cell Death and Disease (2017) 8, e3054; doi:10.1038/cddis.2017.419; published online 21 September 2017

Preeclampsia (PE) is a pregnancy-specific disorder in humans that serves as a predominant contributor to maternal mortality and affects, approximately $2-8 \%$ of pregnancies around the world. ${ }^{1}$ Although the cause and pathophysiology of $\mathrm{PE}$ remain largely unclear, it is generally accepted that the placenta is of great importance in the pathogenesis of PE because removal of the placenta can eradicate clinical symptoms in the patients with $\mathrm{PE}^{2}$ At present, a growing body of evidence indicates that abnormally shallow placentation in early pregnancy is mainly responsible for the onset of $\mathrm{PE}^{3}$ Excessive apoptosis of trophoblast cells, poor invasion of the uterine wall by trophoblasts and impaired remodelling of spiral arteries at the maternal-foetal interface are major abnormal placentation events that are closely related to PE. A wide range of growth factors and hormones are also believed to be involved in the intricate regulation of these events.

Peroxisome proliferator-activated receptors, including PPAR $a$, PPAR $\beta / \delta$ and PPAR $\gamma$, are ligand-activated transcription factors that regulate a number of genes associated with cell differentiation and proliferation. ${ }^{4}$ PPAR $y$ has an important role in the differentiation of complicated trophoblast lineages and normal vascular function. ${ }^{5-7}$ In addition, recent studies have shown that serum concentrations of endogenous activators of PPARy are dramatically decreased in severe $\mathrm{PE}$ patients compared with healthy pregnant women, ${ }^{8}$ and PPARy may serve as a novel therapeutic target for PE. ${ }^{9}$ Nevertheless, the molecular mechanisms of the protective roles of PPARy in PE remain largely unknown.
Angiopoietin-like protein 4 (ANGPTL4), a secretory glycoprotein, is a member of the angiopoietin family. ${ }^{10}$ Previous studies have suggested that ANGPTL4 is a multifunctional factor involved in the regulation of lipid metabolism, wound healing and angiogenesis. ${ }^{11,12}$ Furthermore, it has been reported that activation of PPARy induces the expression and secretion of ANGPTL4. ${ }^{13,14}$ However, the expression and secretion of ANGPTL4 in PE has not been investigated. In addition, it remains unknown whether a regulatory interaction between ANGPTL4 and PPARy exists in PE.

Here, we investigated the effects of the PPARy agonist rosiglitazone on the expression and secretion of ANGPTL4 and the molecular mechanisms underlying these effects in HTR8/SVneo cells, human umbilical vein endothelial cells (HUVECs) and placental explants. Moreover, we explored the expression of ANGPTL4 and PPARy in placental tissue and serum as well as their potential correlation in preeclamptic patients and healthy subjects. To further identify the latent roles of ANGPTL4 and PPARy in PE, a variety of functional studies were performed using cell lines and placental explant models.

\section{Results}

PPARy is indispensable for the rosiglitazone-induced expression and secretion of ANGPTL4. To examine the role of the PPARy agonist rosiglitazone in the expression and secretion of ANGPTL4, HTR8/SVneo cells, HUVECs and placental explants were stimulated with different concentrations $(0,0.25,0.5$ and $1 \mu \mathrm{M})$ of Rosi (rosiglitazone). As shown

\footnotetext{
${ }^{1}$ Department of Obstetrics and Gynecology, Ren Ji Hospital, School of Medicine, Shanghai Jiao Tong University, Shanghai, China and ${ }^{2}$ Shanghai Key Laboratory of Gynecologic Oncology, Ren Ji Hospital, School of Medicine, Shanghai Jiao Tong University, Shanghai, China

*Corresponding author: J Lin, Department of Obstetrics and Gynecology, Ren Ji Hospital, School of Medicine, Shanghai Jiao Tong University, 160 Pujian Road, Shanghai 200127, China. Tel/Fax: +86 21 68383073; E-mail: linjianhrj@126.com

Received 07.5.17; revised 06.7.17; accepted 18.7.17; Edited by Y Shi
} 
a

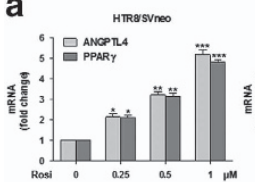

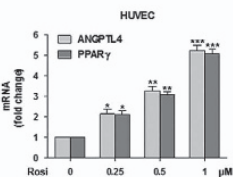

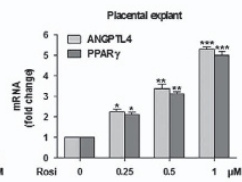

b
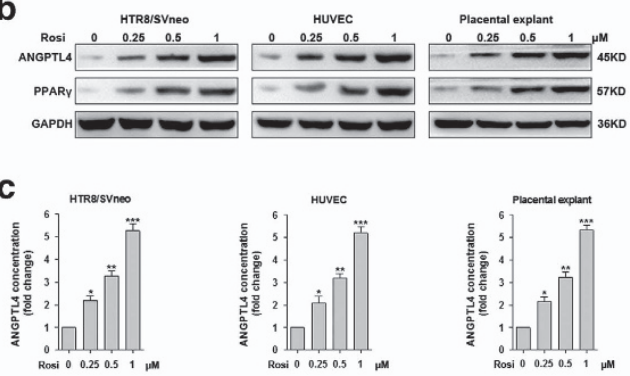

e
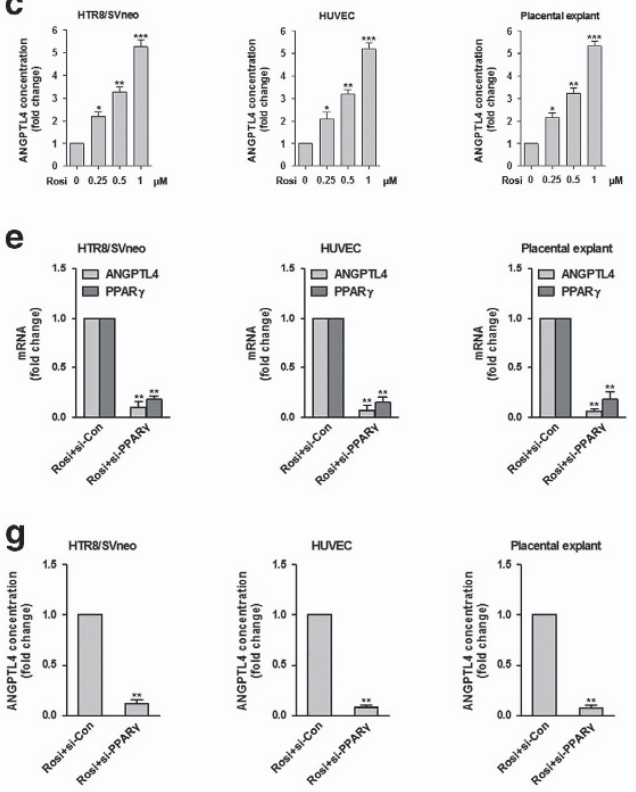

Figure 1 Rosiglitazone stimulates the expression and secretion of ANGPTL4 via PPAR $\gamma$. (a-c) HTR8/SVneo cells, HUVECs and placental explants were stimulated with Rosi (rosiglitazone) $(0,0.25,0.5$ and $1 \mu \mathrm{M})$ for $18 \mathrm{~h}$. The expression of PPAR $\gamma$ and ANGPTL4 and the secretion of ANGPTL4 were determined by quantitative real-time PCR (qRTPCR), western blot and enzyme-linked immunosorbent assay (ELISA). The data are shown as the means \pm S.E.M. ${ }^{*} P<0.05,{ }^{* \star} P<0.01$ and ${ }^{* \star *} P<0.001$ compared with control. (d) HUVECs, HTR8/SVneo cells and placental explants were stimulated with different concentrations $(0$ and $1 \mu \mathrm{M})$ of rosiglitazone for $18 \mathrm{~h}$, and then stained to detect the expression of PPAR $\gamma$ and ANGPTL4 by immunofluorescence. (e-h) HTR8/SVneo cells, HUVECs and placental explants were transfected with control siRNA (si-Con) or PPAR $\gamma$ siRNA (si-PPAR $\gamma$ ), and then treated with $1 \mu \mathrm{M}$ rosiglitazone for $18 \mathrm{~h}$. The expression of PPAR $\gamma$ and ANGPTL4 and the secretion of ANGPTL4 were evaluated by qRT-PCR, western blot analysis, ELISA and immunofluorescence. The data are shown as the means \pm S.E.M. ${ }^{* *} P<0.01$ relative to corresponding control

in Figures 1a-c and Supplementary Figure 1a, rosiglitazoneinduced ANGPTL4 mRNA and protein expression and its secretion in a concentration-dependent manner. Rosiglitazone also induced the mRNA and protein expression of PPARy in a similar manner (Figures 1a-c). The rosiglitazoneinduced expression of ANGPTL4 and PPARy was also demonstrated by immunofluorescence staining (Figure 1d).

To further determine the role of PPARy in the rosiglitazoneinduced expression and secretion of ANGPTL4, HTR8/SVneo cells, HUVECs and placental explants were transfected with control siRNA (si-Con) or PPARy siRNA (si-PPAR $y$ ) and treated with $1 \mu \mathrm{M}$ rosiglitazone. The results showed that silencing of PPARy expression inhibited the effects of rosiglitazone on ANGPTL4 protein and mRNA expression as well as its secretion (Figures $1 \mathrm{e}-\mathrm{g}$ and Supplementary Figure 1b). The same results were confirmed in the cell lines and explants through immunofluorescence staining (Figure $1 \mathrm{~h}$ and Supplementary Figure 1c). These data suggest that
PPAR $y$ is indispensable for the rosiglitazone-induced expression and secretion of ANGPTL4.

ANGPTL4 is a direct transcriptional target of PPAR $\boldsymbol{y}$. Activated PPARy regulates gene expression via heterodimerizing with retinoid $X$ receptors (RXRs) and binding to the peroxisome proliferator-responsive element (PPRE) of target genes. ${ }^{15}$ Given that PPARy is implicated in the regulation of ANGPTL4 expression and secretion, we speculated that a PPRE likely exists in the promoter region of ANGPTL4 for its transactivation. To test this hypothesis, we analysed the human ANGPTL4 5'-flanking region and identified three putative PPREs, namely PPRE1 (-1822/-1808), PPRE2 (-809/ - 795) and PPRE3 (-233/-209), located upstream of the ANGPTL4 transcription start site (Figure 2a). Next, to determine whether PPARy binds to these regions, a chromatin immunoprecipitation (ChIP) assay was performed 


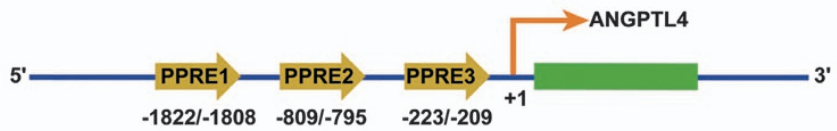

b

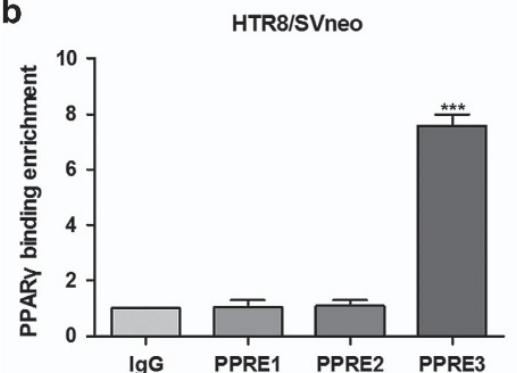

C

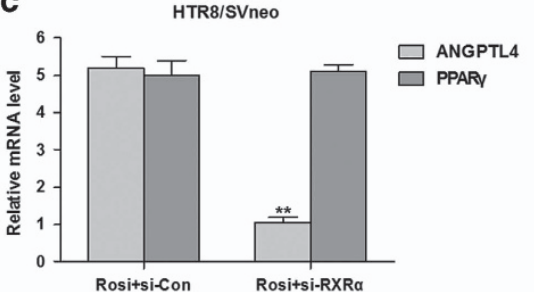

d

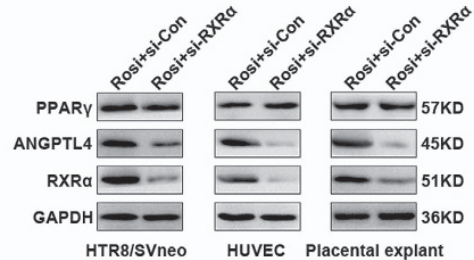

f

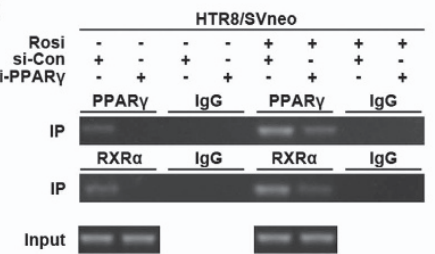

HUVEC
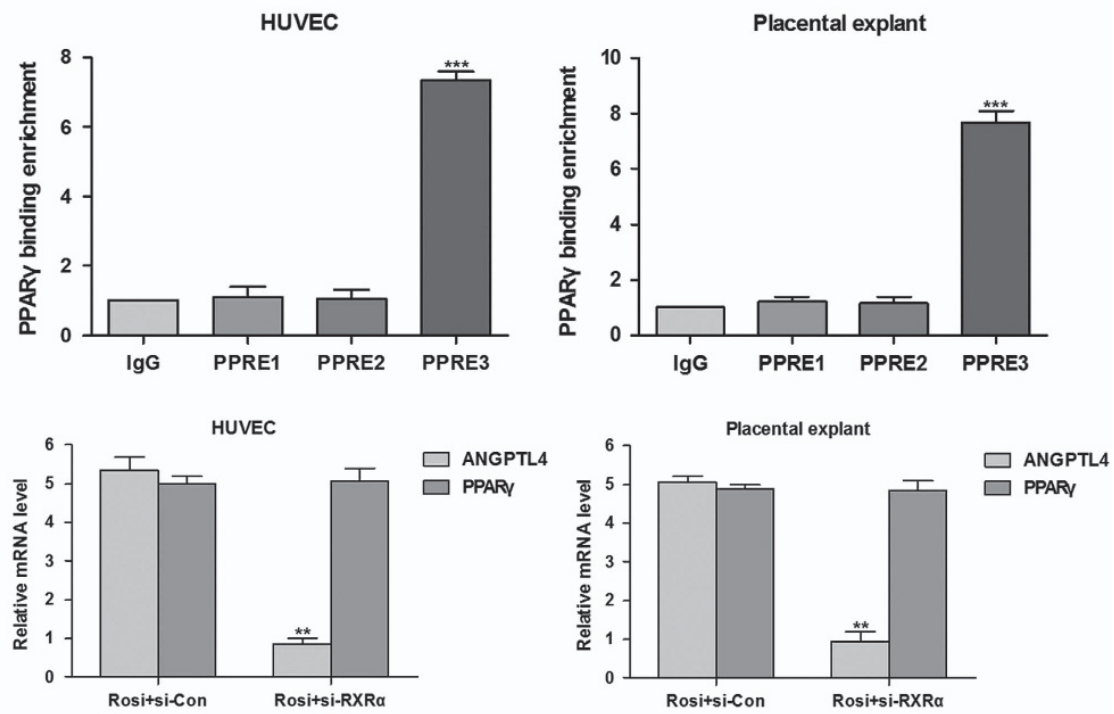

e
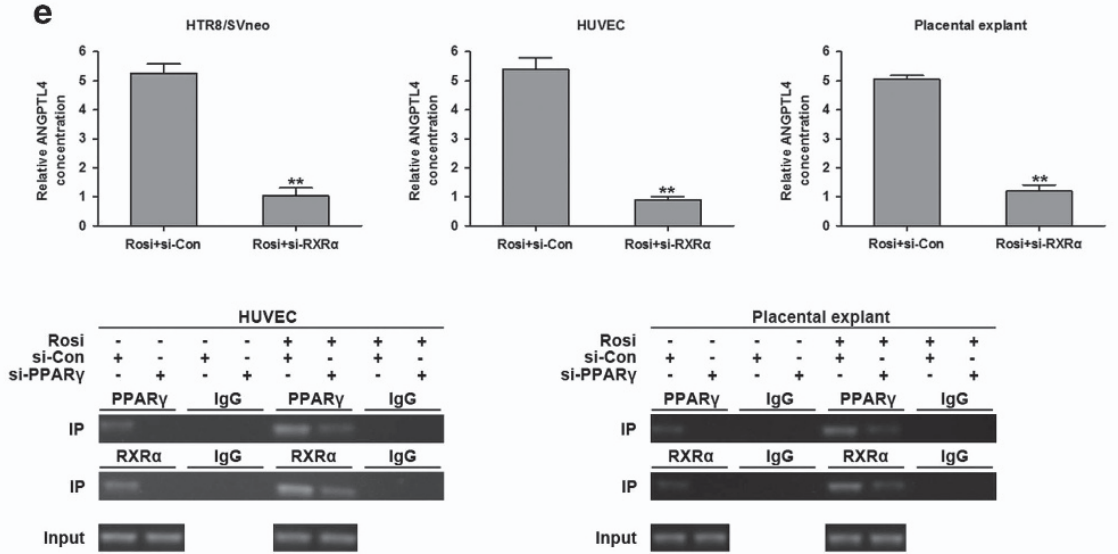

Figure 2 The PPAR $\gamma$ agonist induces ANGPTL4 expression and secretion by promoting the binding of the PPAR $\gamma /$ RXR $\alpha$ heterodimer to its promoter. (a) Schematic representation of the ANGPTL4 gene promoter showing the location of the potential peroxisome proliferator-responsive elements (PPREs). (b) Chromatin immunoprecipitation (ChIP) assays were performed in HTR8/SVneo cells, HUVECs and placental explants using antibodies against PPAR $\gamma$ or lgG. Purified DNA was detected by qRT-PCR using primer sets specific to PPRE1-PPRE3 of ANGPTL4. The data are shown as the means \pm S.E.M. ${ }^{* \star *} P<0.001$ relative to $\operatorname{lgG}$. (c-e) HTR8/SVneo cells, HUVECs and placental explants were transfected with control siRNA (si-Con) or RXR $\alpha$ siRNA (si-RXR $\alpha$ ) and then stimulated with $1 \mu \mathrm{M}$ rosiglitazone for $18 \mathrm{~h}$. The mRNA and protein expression of PPAR $\gamma$ and ANGPTL4, the protein expression of RXR $\alpha$ and the secretion of ANGPTL4 were detected via qRT-PCR, western blot analysis and ELISA. The data are shown as the means \pm S.E.M. ${ }^{* *} P<0.01$ versus control. (f) HUVECs, HTR8/SVneo cells and placental explants were transfected with si-Con or si-PPAR $\gamma$ and then treated with or without $1 \mu \mathrm{M}$ rosiglitazone for $18 \mathrm{~h}$. ChIP assays were accomplished with specific antibodies against PPAR $\gamma$ and RXR $\alpha$

in HTR8/SVneo cells, HUVECs and placental explants. The results indicated that PPAR $y$ binds to PPRE3, located at $-233 /-209$ (Figure 2b).

The PPAR $y /$ retinoid X receptor a (PPAR $y / \mathrm{RXR} a)$ heterodimer is required for placental development. ${ }^{16}$ To investigate whether RXRa is involved in the rosiglitazone-induced expression and secretion of ANGPTL4, HTR8/SVneo cells, HUVECs and placental explants were transfected with control siRNA (si-Con) or RXRa siRNA (si-RXRa) and treated with rosiglitazone. As shown in Figures $2 \mathrm{c}-\mathrm{e}$, silenced $\mathrm{RXR} a$ expression abolished the effects of rosiglitazone on ANGPTL4 protein and mRNA expression, as well as its secretion.
Surprisingly, the rosiglitazone-induced PPAR $y$ protein and mRNA expression were not affected by treatment with si$\mathrm{RXRa}$ (Figures $2 \mathrm{c}$ and $\mathrm{d}$ ). These results demonstrate that $\mathrm{RXR} a$ is a prerequisite for rosiglitazone-induced ANGPTL4 expression. Subsequently, to further determine whether RXR $a$ also binds to the PPRE3 in ANGPTL4 promoter region, ChIP assays were carried out in HTR8/SVneo cells, HUVECs and placental explants. The data showed that both PPAR $y$ and RXRa bound to PPRE3 in the ANGPTL4 promoter (Figure 2f). Together, these results indicate that rosiglitazone stimulates ANGPTL4 expression via regulating the binding of the PPAR $y /$ RXRa heterodimer to its promoter. 
The expression and circulating activators of PPAR $y$ and the expression and secretion of ANGPTL4 are decreased in PE. As shown in Supplementary Table 1, there were no obvious differences in body mass index, age, gestational age and infant birth weight between normal pregnant $(n=30)$ and preeclamptic women $(n=30)$ enroled in this study. Next, to assess the physiological significance of PPARy and ANGPTL4 in placental development, we detected their mRNA and protein expression among placental tissues by western blot, quantitative real-time PCR (qRT-PCR) and
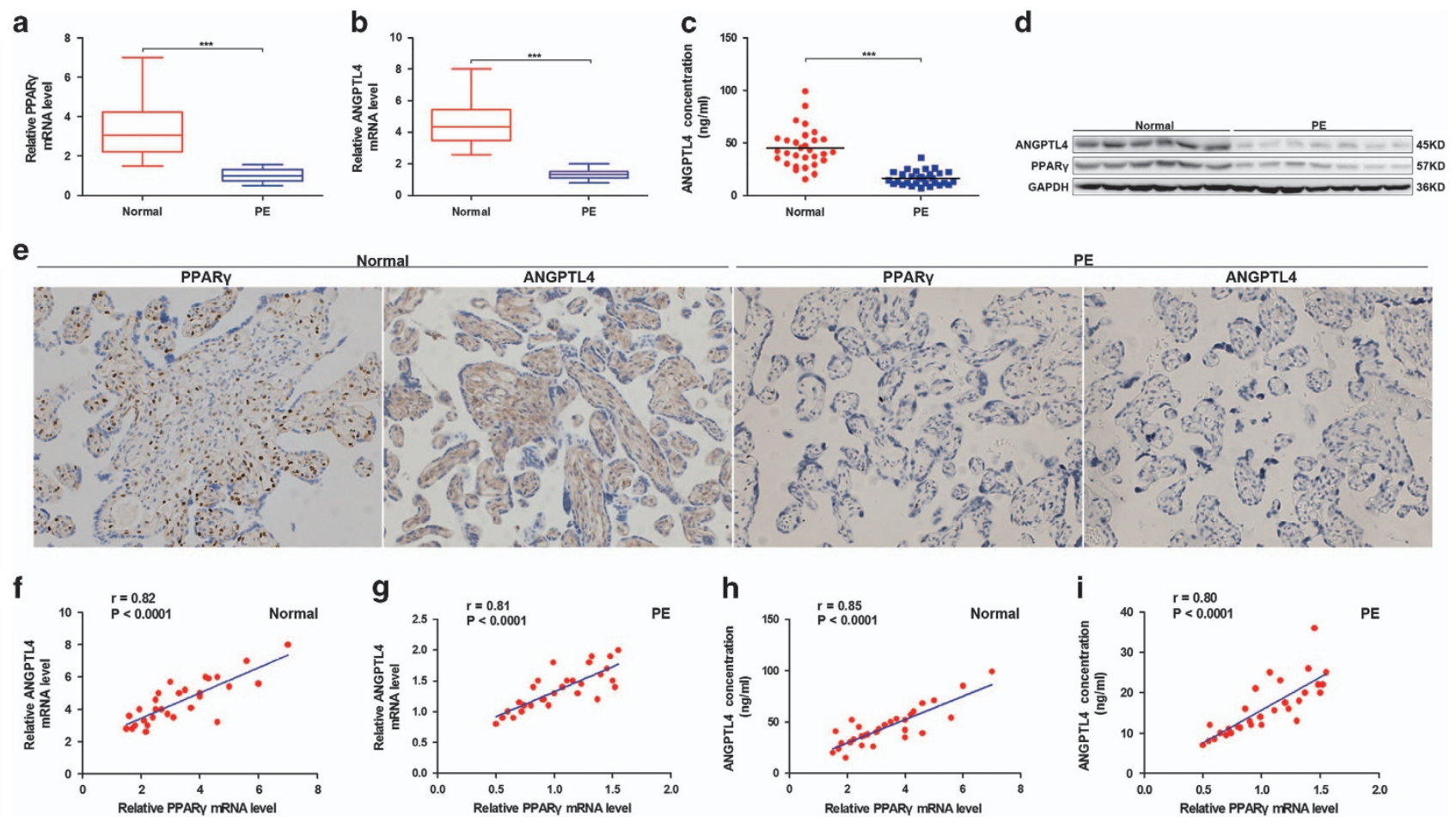

Normal ANGPTL4
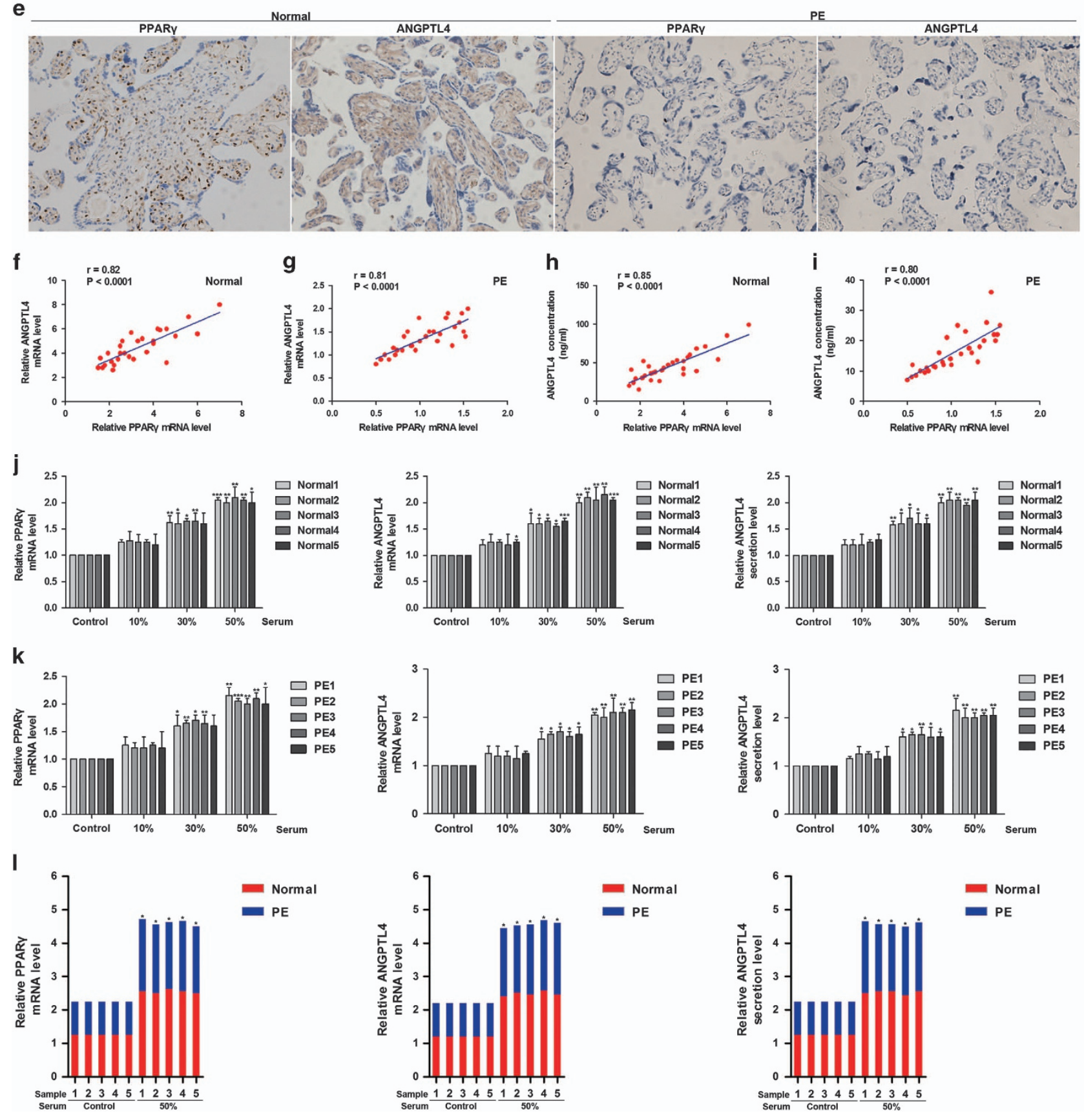
immunohistochemistry (IHC) analyses. The results showed that the mRNA and protein expression levels of PPARy and ANGPTL4 were significantly decreased in PE samples compared with normal controls (Figures $3 a, b, d$ and e). The secretion of ANGPTL4 in the serum was also decreased in PE subjects compared with normal controls $(16.2 \pm 1.2$ versus $44.9 \pm 3.4 \mathrm{ng} / \mathrm{ml}$, ${ }^{* *} P<0.001$ ) (Figure $3 \mathrm{c}$ ).

Because the PPARy agonist rosiglitazone induces the mRNA and protein expression of PPAR $y$ in a concentrationdependent way in placental explants, we speculated that circulating activators of PPAR $y$ were decreased in PE subjects compared with normal pregnant women. To prove this hypothesis, placental explants were treated with serum from normal controls $(n=5)$ and PE subjects $(n=5)$. The mRNA expression of PPARy and ANGPTL4 as well as the secretion of ANGPTL4 were upregulated by serum from both PE and control subjects in a concentration-dependent manner (Figures 3j and k) and increased in normal pregnant women compared with PE (Figure 31 and Supplementary Figure 2). In addition, PPARy mRNA was significantly reduced in preeclamptic placentae compared with normal placentae (Figure 3a). Based on the above results, our hypothesis that circulating activators of PPARy in PE are decreased may be correct.

Next, correlation analysis revealed a positive relationship between the mRNA level of PPAR $y$ and the mRNA level and secretion of ANGPTL4 in PE $(n=30)$ and normal pregnant women $(n=30)$ (Figures $3 f-i)$, suggesting a functional interaction between PPAR $y$ and ANGPTL4 in vivo. Given that serum from PE and control subjects stimulated PPAR $y$ mRNA expression in a concentration-dependent manner, the mRNA level of PPARy in placental tissues should be an indicator of circulating activators of PPARy in serum. Together with the results shown in Figures $3 f-i$, we hypothesised that circulating PPARy activators positively correlate with the secretion of ANGPTL4 in normal pregnant and PE women. Taken together, the expression and circulating activators of PPAR $y$ and the expression and secretion of ANGPTL4 are decreased in PE, and the reduced expression and secretion levels of ANGPTL4 may result from a decrease in circulating activators of PPAR $y$ in serum.

ANGPTL4 mediates rosiglitazone-induced trophoblast cell survival and proliferation. Excessive apoptosis of trophoblast cell is a major abnormal placentation event involved in PE. ${ }^{3}$ Oxidative stress is responsible for increased trophoblast cell apoptosis. ${ }^{17}$ Therefore, we determined the effects of ANGPTL4 and PPARy on trophoblast cell survival. HTR8/SVneo cells transfected with control siRNA (si-Con) or
ANGPTL4 siRNA (si-ANGPTL4) were treated with $150 \mu \mathrm{M}$ hydrogen peroxide and $1 \mu \mathrm{M}$ rosiglitazone for 48 hours. Controls were stimulated with $150 \mu \mathrm{M}$ hydrogen peroxide and $100 \mathrm{nM}$ recombinant human ANGPTL4 (rhANGPTL4). Subsequently, terminal deoxynucleotidyl transferase-mediated deoxyuridine triphosphate nick end labelling (TUNEL) assays were performed to determine the rate of cellular apoptosis. Rosiglitazone and rhANGPTL4 dramatically inhibited trophoblast cell apoptosis induced by hydrogen peroxide, and the deletion of ANGPTL4 abolished the antiapoptotic effects of rosiglitazone (Figure 4a and Supplementary Figure 3). At the same time, the expression of molecular markers associated with apoptosis, such as Bax, cleaved PARP, caspase-9, cleaved caspase-9, caspase-3 and cleaved caspase-3, was also reduced in cells treated with rosiglitazone and rhANGPTL4 compared with the control (Figures $4 \mathrm{~b}$ and $\mathrm{c}$ ). Furthermore, the expression of $\mathrm{CK} 7$ (a marker of trophoblast), PPAR $\gamma$, ANGPTL4 and caspase-3 was assessed through $\mathrm{IHC}$ in preeclamptic placentae and normal controls. The results indicated that caspase-3 expression was increased in preeclamptic placentae with lower PPARy and ANGPTL4 expression compared with normal placentae (Figure $4 \mathrm{~g}$ ).

Next, the roles of ANGPTL4 and PPARy in trophoblast cell proliferation were assessed using a cell proliferation assay. Similarly, rosiglitazone and rhANGPTL4 significantly promoted trophoblast cell proliferation compared with controls, and ablation of ANGPTL4 blocked the effects of rosiglitazone (Figure 4d). In addition, the expression of cell proliferationrelated genes, such as Cyclin D1, Bcl-2, c-Myc and pHH3, was clearly upregulated in cells treated with rosiglitazone and rhANGPTL4 compared with controls (Figures $4 e$ and f). Consistent with these results, decreased expression of PPARy, ANGPTL4 and cyclin D1 was observed in PE placentae compared with controls by IHC (Figure $4 \mathrm{~g}$ ). In conclusion, these data indicate that ANGPTL4 is essential for the PPARy agonist-induced survival and proliferation of trophoblasts.

\footnotetext{
ANGPTL4 is important for the migration and invasion of trophoblast cell and placental explant outgrowth induced by the PPARy agonist. To elucidate the roles of ANGPTL4 and PPARy in trophoblast cells migration and invasion, transwell invasion and wound-healing assays were executed in HTR8/SVneo cells transfected with si-ANGPTL4 or si-Con and control cells. Rosiglitazone and rhANGPTL4 exhibited similar roles in the migration and invasion of trophoblast cells compared with control cells, and silenced ANGPTL4 abolished the effects of rosiglitazone (Figures 5a,b and d).
}

Figure 3 The expression and secretion of ANGPTL4 are decreased and positively correlated with the expression of PPAR $\gamma$ in PE. (a, $\mathbf{b})$ The mRNA levels of PPAR $\gamma$ and ANGPTL4 in placental tissues were detected in PE $(n=30)$ and normal controls $(n=30)$ by qRT-PCR. ${ }^{* \star *} P<0.001$ against normal controls. (c) The secretion of ANGPTL4 in the serum was measured among PE $(n=30)$ and normal controls $(n=30)$ by ELISA. ${ }^{* \star \star} P<0.001$ compared with normal controls. (d) The expression of ANGPTL4 and PPAR in placental tissues was determined by western blot analysis in seven PE subjects and six normal controls randomly selected from 60 placental tissues. (e) Immunohistochemistry $(\mathrm{IHC})$ was performed to analyse the expression of PPAR $\gamma$ and ANGPTL4 in PE subjects $(n=30)$ and normal controls $(n=30)$. (f-i) The relationship between the mRNA expression of PPAR $\gamma$ and the mRNA and secretion of ANGPTL4 was analysed in PE subjects $(n=30)$ and normal controls $(n=30)$. (j, k) Placental explants were stimulated with serum from PE subjects $(n=5)$ and normal controls $(n=5)$ randomly selected from 60 blood samples. PPAR $\gamma$ and the mRNA and secretion of ANGPTL4 were assessed by qRTPCR and ELISA. The data are shown as the means \pm S.E.M. ${ }^{\star} P<0.05$, ${ }^{\star \star} P<0.01$ and ${ }^{\star * \star} P<0.001$ compared with corresponding control. (I) The combined data from (j, $\mathbf{k}$ ) was analysed. The data are shown as the means. ${ }^{*} P<0.05$ compared with $\mathrm{PE}$ 
a
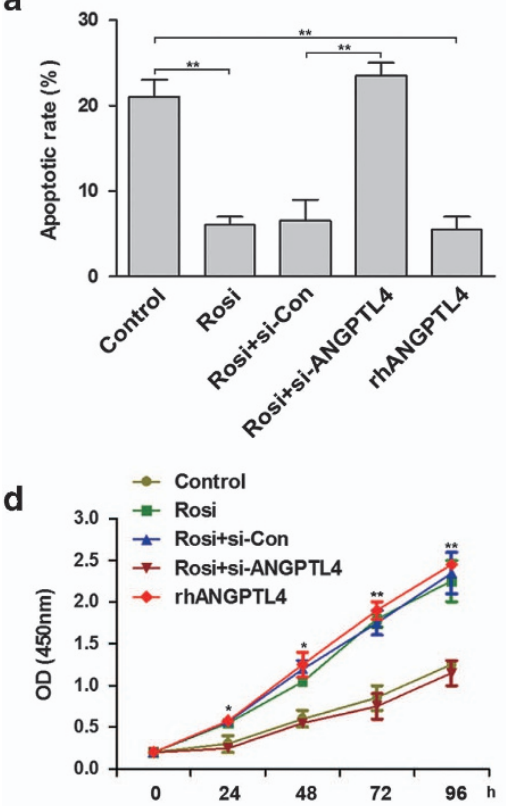

b

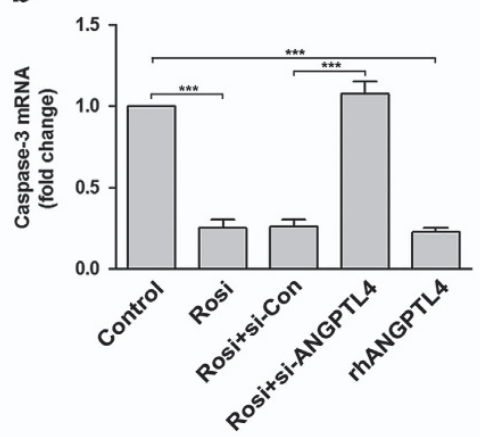

e

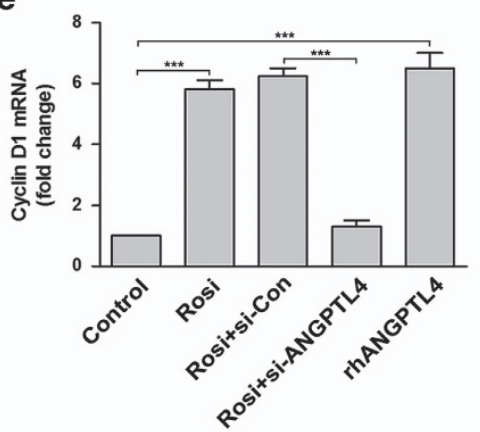

c

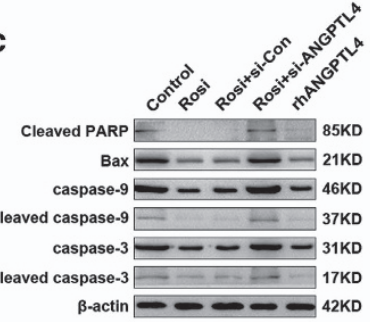

f

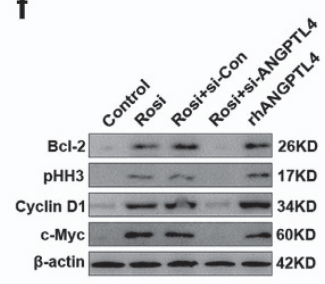

g CK7 PPARY ANGPTL4 Caspase-3 Cyclin D1

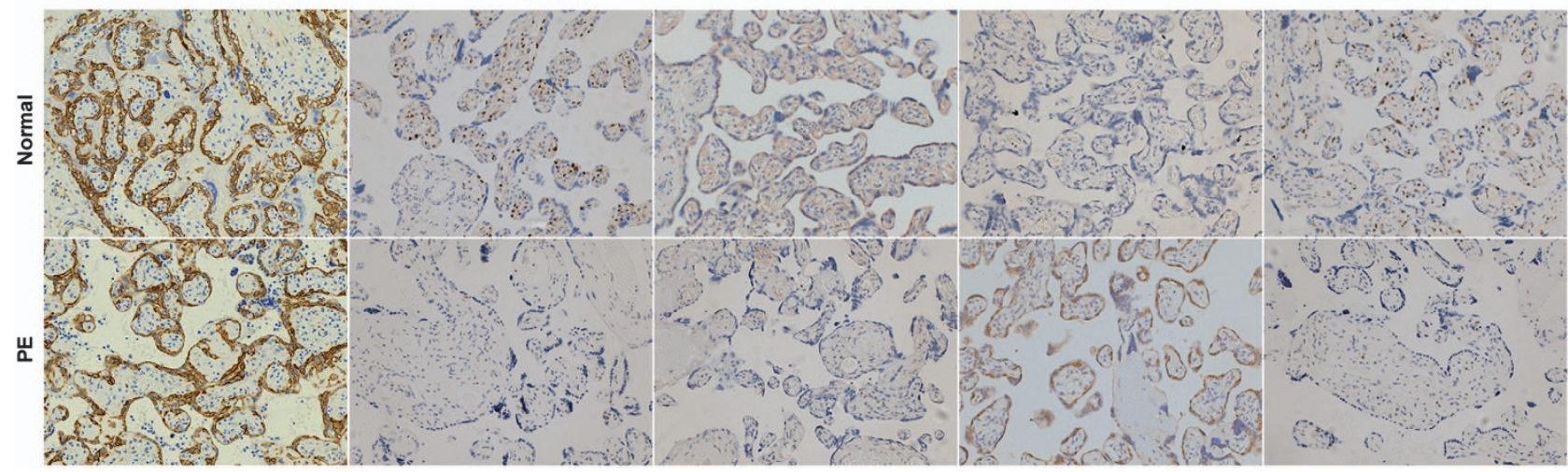

Figure 4 ANGPTL4 is essential for rosiglitazone-induced trophoblast cell survival and proliferation. (a-c) HTR8/SVneo cells transfected with control siRNA (si-Con) or ANGPTL4 siRNA (si-ANGPTL4) were stimulated with $1 \mu \mathrm{M}$ rosiglitazone in the presence of $150 \mu \mathrm{M}$ hydrogen peroxide. Control cells were treated with $1 \mu \mathrm{M}$ rosiglitazone or $100 \mathrm{nM}$ recombinant human ANGPTL4 (rhANGPTL4) in the presence of $150 \mu \mathrm{M}$ hydrogen peroxide. A TUNEL assay was performed to evaluate the rate of cellular apoptosis. Caspase- 3 expression was detected by qRT-PCR, and the expression of caspase-9, cleaved caspase-9, caspase-3, cleaved caspase-3, cleaved PARP and Bax was measured via western blot analysis. The data are shown as the means \pm S.E.M. ${ }^{* \star} P<0.01,{ }^{* \star \star} P<0.001$ compared with control or si-Con. (d-f) HTR8/SVneo cells transfected with si-Con or si-ANGPTL4 were stimulated with $1 \mu \mathrm{M}$ rosiglitazone. Control cells were treated with $1 \mu \mathrm{M}$ rosiglitazone or $100 \mathrm{nM}$ rhANGPTL4. A CCK-8 assay was performed to examine cell proliferation. Cyclin D1 mRNA was assessed by qRT-PCR, and the expression of Bcl-2, pHH3, Cyclin D1 and c-Myc was determined by western blot analysis. The data are shown as the means \pm S.E.M. ${ }^{*} P<0.05$, ${ }^{*} P<0.01$ and ${ }^{* \star *} P<0.001$ against control or si-Con. (g) The expression of CK7, PPAR $\gamma$, ANGPTL4, Cyclin D1 and caspase-3 in placental tissues was further analysed by IHC in PE subjects $(n=30)$ and normal controls $(n=30)$. Representative images were captured at $\times 200$ magnification

Figure 5 ANGPTL4 meditates PPAR $\gamma$ agonist-induced migration and invasion of trophoblast cells and outgrowth of placental explants. (a-e) HTR8/SVneo cells transfected with si-Con or si-ANGPTL4 were stimulated with $1 \mu \mathrm{M}$ rosiglitazone. Control cells were treated with $1 \mu \mathrm{M}$ rosiglitazone or $100 \mathrm{nM}$ rhANGPTL4. Transwell invasion and woundhealing assays were performed to assess the invasive and migratory abilities of these cells. The expression of MMP-2, MMP-9, TIMP-1 and TIMP-2 was tested via western blot analysis. The data are shown as the means \pm S.E.M. ${ }^{*} P<0.05$, ${ }^{\star \star} P<0.01$ and ${ }^{* \star *} P<0.001$ versus control or si-Con. (f-h) Placental explants transfected with si-Con or si-ANGPTL4 were treated with $1 \mu \mathrm{M}$ rosiglitazone. Control explants were stimulated with $1 \mu \mathrm{M}$ rosiglitazone or $100 \mathrm{nM}$ rhANGPTL4. Placental explant outgrowth was measured to further assess extravillous cytotrophoblast (EVT) migration and invasion. Representative images of placental explants are shown. The expression of MMP-9 and MMP-2 was measured via gelatin zymography, and the expression of TIMP-1 and TIMP-2 was determined by western blot analysis. The data are shown as the means \pm S.E.M. ${ }^{* \star} P<0.01$, ${ }^{* * *} P<0.001$ compared with control or si-Con. (i) The expression of PPAR $\gamma$, ANGPTL4, MMP-2 and MMP-9 in placental tissues was determined by IHC in PE subjects ( $n=30$ ) and normal controls $(n=30)$. Representative images were captured at $\times 200$ magnification 
To determine the mechanisms by which rosiglitazone and ANGPTL4 act synergistically to promote trophoblast cells migration and invasion, we investigated their roles in matrix metalloproteinase-2 (MMP-2) and matrix metalloproteinase-9 (MMP-9) expression. Compared with control cells, rosiglitazone and rhANGPTL4 significantly increased the expression

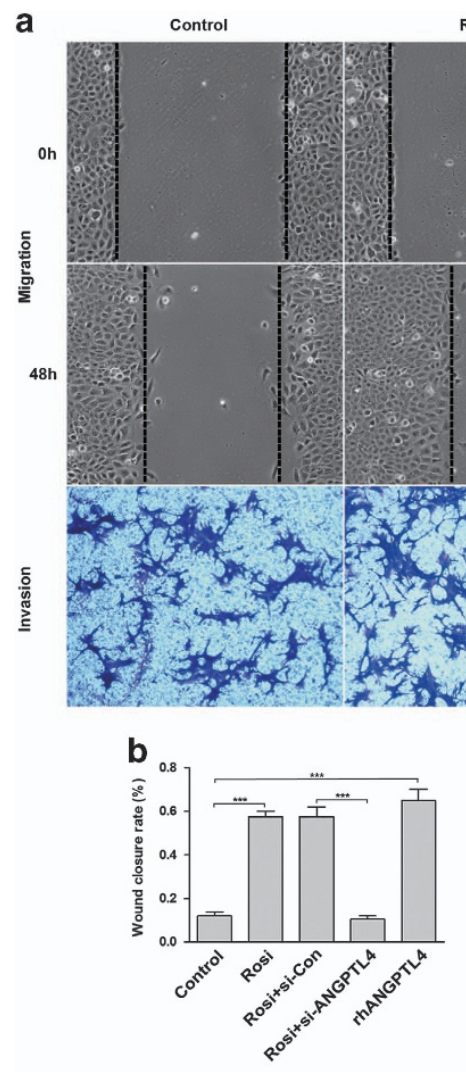

Rosi

Rosi+si-Con

Rosi+si-ANGPTL4

rhANGPTL4
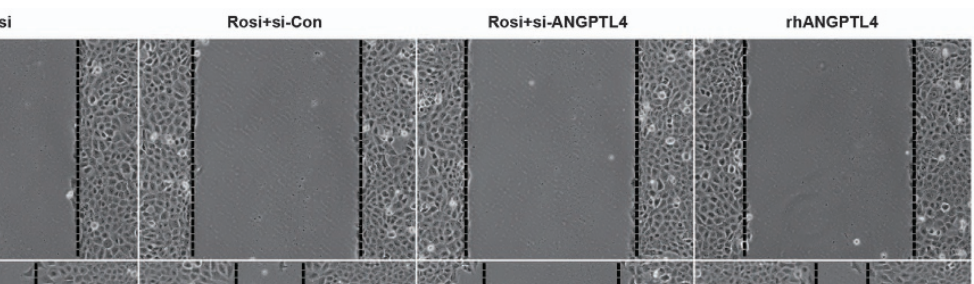

f

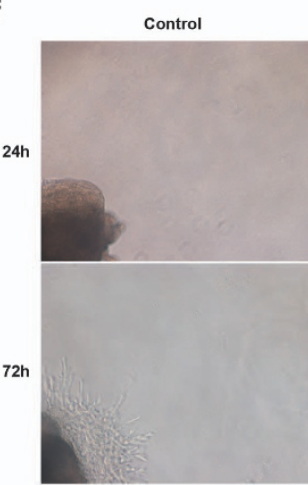

c

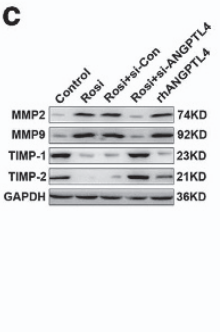

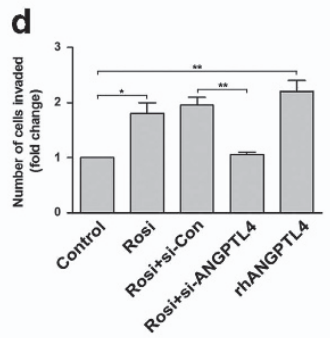

e

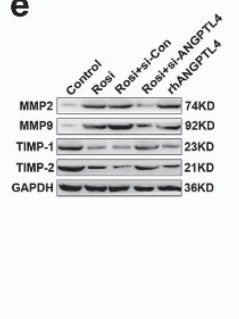

Rosi

Rosi+si-Con

Rosi+si-ANGPTL4 rhANGPTL4

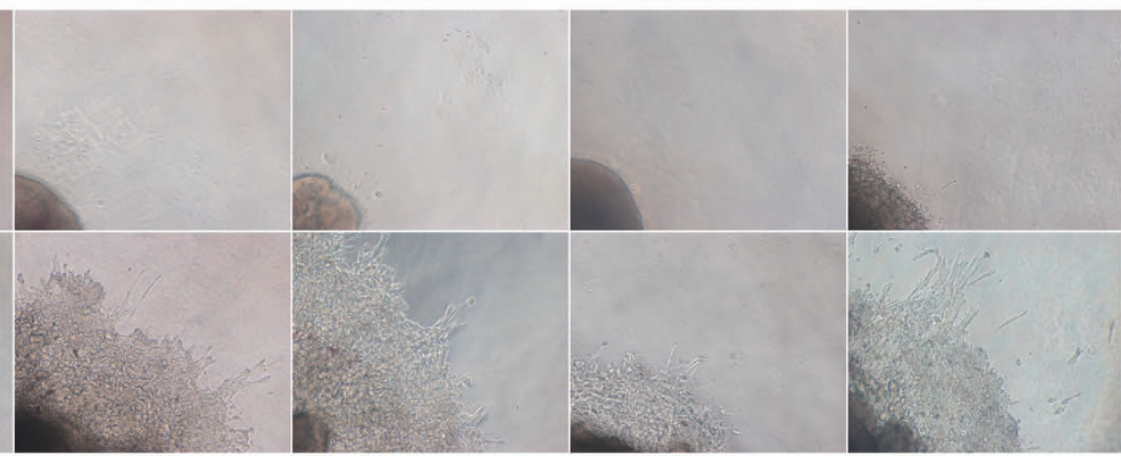

g

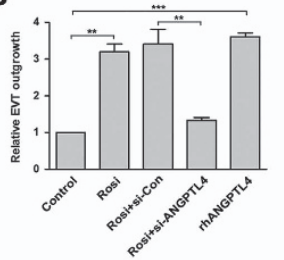

h

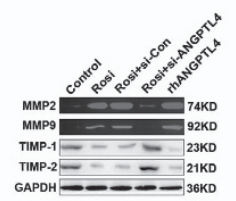

i

PPARY

ANGPTL4

MMP2

MMP9

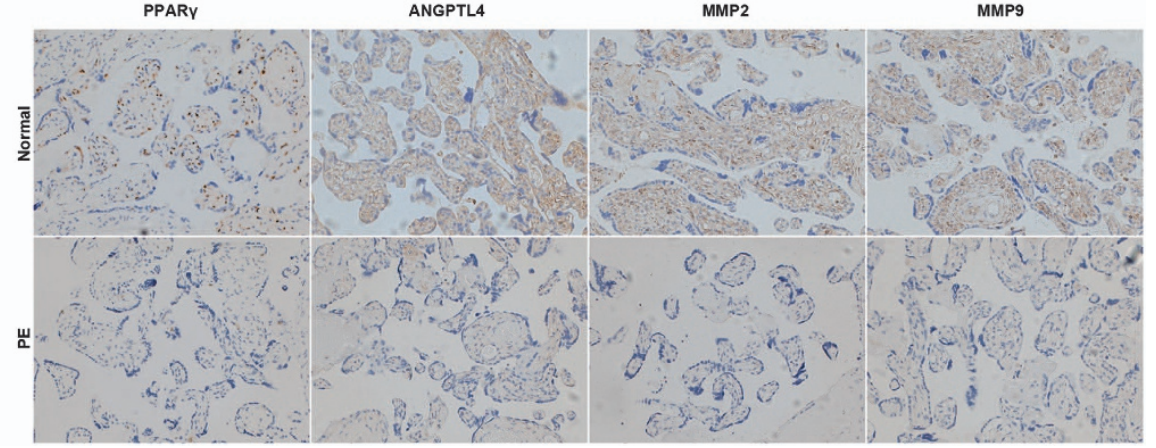


of MMP-2 and MMP-9, accompanying with decreased tissue inhibitor of metalloproteinase-1 (TIMP-1) and tissue inhibitor of metalloproteinase-2 (TIMP-2) expression (the tissue inhibitors of MMP-9 and MMP-2) (Figures 5c and e).

To further verify the physiological roles of PPARy and ANGPTL4 in trophoblast cell invasion and migration, placenta villous explants were performed. As expected, rosiglitazone and rhANGPTL4 markedly promoted the outgrowth of placenta villous explants compared with the controls, and deletion of ANGPTL4 abolished the effects of rosiglitazone (Figures $5 f$ and $g$ ). The expression of MMP-2 and MMP-9 was also measured by gelatin zymography, which indicated that their expression, as well as that of TIMP-1 and TIMP-2 was consistent with previous results (Figure 5h). Furthermore, IHC analysis revealed decreased expression of MMP-2 and MMP-9 in preeclamptic placentae $(n=30)$ compared with normal placentae $(n=30)$ (Figure $5 \mathrm{i})$. These observations collectively demonstrate that ANGPTL4 mediates the PPARy agonist-induced migration and invasion of trophoblast cells and placental explant outgrowth via regulating MMP-2 and MMP-9.

ANGPTL4 is involved in PPARy agonist-induced angiogenesis. Limited remodelling of the spiral arteries is a main pathological feature of $\mathrm{PE}^{3}$ To explore the roles of PPARy and ANGPTL4 in angiogenesis, a tube formation assay was performed on HUVECs transfected with si-Con or si-ANGPTL4 and control cells. Rosiglitazone and rhANGPTL4 distinctly increased the total tube length, total number of tubes and total branch points compared with controls, but depletion of ANGPTL4 eliminated the effects of rosiglitazone in angiogenesis (Figures $6 \mathrm{a}$ and b). Moreover, the expression and secretion of vascular endothelial growth factor (VEGF) was examined via qRT-PCR, western blot and ELISA, which yielded results similar to those from the tube formation assay (Figure 6c). Eventually, the expression of CD31 (an indicator of vascularity) and VEGF in placental tissues was evaluated by $\mathrm{IHC}$, which indicated their decreased expression in preeclamptic placentae $(n=30)$ compared with normal placentae $(n=30)$ (Figure $6 \mathrm{~d})$. All in all, these data suggest that ANGPTL4 is an important mediator of PPARy agonist-induced angiogenesis.

\section{Discussion}

In the present study, we show that PPARy is a prerequisite for the PPARy agonist-induced expression and secretion of ANGPTL4 in HTR8/SVneo cells, HUVECs and placental explants in a concentration-dependent manner. We also identify ANGPTL4 as a direct transcriptional target of PPARy and demonstrate for the first time that decreased expression and secretion of ANGPTL4 may result from a decrease in circulating PPARy activators in preeclamptic patients compared with normal pregnant subjects. In addition, we provide evidence that ANGPTL4 mediates the facilitative effects of PPARy agonist-induced survival, proliferation, migration and invasion in trophoblast cells, as well as outgrowth in placental explants and angiogenesis in HUVECs. In conclusion, these findings indicate that ANGPTL4 is involved in the protective effects of PPARy activators on the pathogenesis of PE (Figure 7).

PPARs are ligand-activated transcriptional factors that regulate many genes associated with cell proliferation and differentiation. ${ }^{4}$ PPAR $y$ is crucial for the differentiation of intricate trophoblast lineages and normal vascular function. ${ }^{5-7}$ Moreover, previous studies have demonstrated that ANGPTL4 serves as a multifunctional factor involved in the regulation of lipid metabolism, wound healing and angiogenesis. ${ }^{11,12}$ However, it is not clear whether the expression of ANGPTL4 is regulated by PPARy agonists in HTR8/SVneo cells, HUVECs and placental explants. Our study demonstrates that the expression and secretion of ANGPTL4 in these cells and placental explants are significantly upregulated in a dose-dependent manner by the PPAR $y$ agonist rosiglitazone. We also found that PPAR $y$ is induced by rosiglitazone in the same manner and is indispensable for the rosiglitazoneinduced expression and secretion of ANGPTL4. Our results are similar to those reported previously in which rosiglitazone was determined to increase the expression of PPARy and ANGPTL4. ${ }^{18,19}$ It has been reported that ANGPTL4 is a target gene of PPAR $\gamma,{ }^{20}$ and ligand-activated PPAR $y$ regulates the expression of genes via binding to the PPRE of target genes as a heterodimer with RXRs. ${ }^{15}$ Therefore, additional studies were performed to elucidate the molecular mechanisms implicated in ANGPTL4 expression induced by rosiglitazone. We provide evidence in HTR8/SVneo cells, HUVECs and placental explants that RXRa is required for the rosiglitazoneinduced expression and secretion of ANGPTL4. In addition, results from our ChIP assay also indicate that the PPAR $y$ / $\mathrm{RXR} a$ heterodimer activates ANGPTL4 transcription by directly binding to PPRE3, located at $-233 /-209$ in its promoter.

PPARy is required for normal placental development, and its activation regulates trophoblast differentiation and invasion. ${ }^{7,21,22}$ Previous studies have shown that ANGPTL4 is involved in the regulation of lipid metabolism, wound healing and angiogenesis. ${ }^{11,12}$ Nevertheless, the expression of PPARy and ANGPTL4 and their correlation are not well characterised in PE and normal pregnancies. We demonstrated in placental tissues and serum that the expression of PPAR $y$ and the expression and secretion of ANGPTL4 were significantly reduced in PE compared with normal control subjects. Our findings that PPARy expression is decreased in PE subjects are supported by those described previously. ${ }^{2}$ In addition, the present study showed that the PPAR $y$ mRNA level is positively correlated with the mRNA level and secretion of ANGPTL4 among normal controls and PE women. A recent study showed that PPARy activators exist in serum obtained from normal pregnant women and upregulate the expression of PPARy. ${ }^{23}$ This prompted us to investigate the level of PPARy activators in the serum among $\mathrm{PE}$ and normal control subjects. Placental explants treated with serum from five normal and five PE women exhibited upregulated expression of PPARy and ANGPTL4 in a concentration-dependent manner. These results indicate that PPARy expression in placental tissue reflects the serum levels of corresponding PPARy activators in normal controls and PE subjects. Taken together, our findings suggest that the levels of PPARy activators in the serum are decreased in women with PE. It 


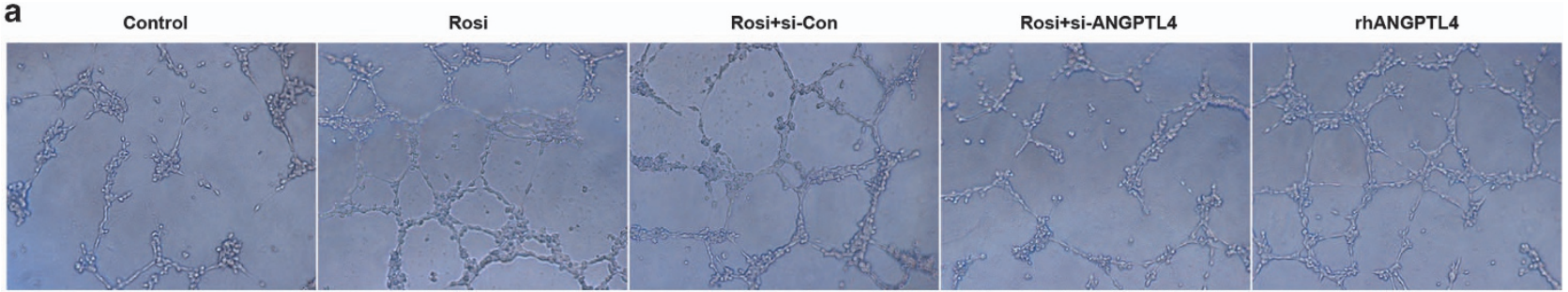

b
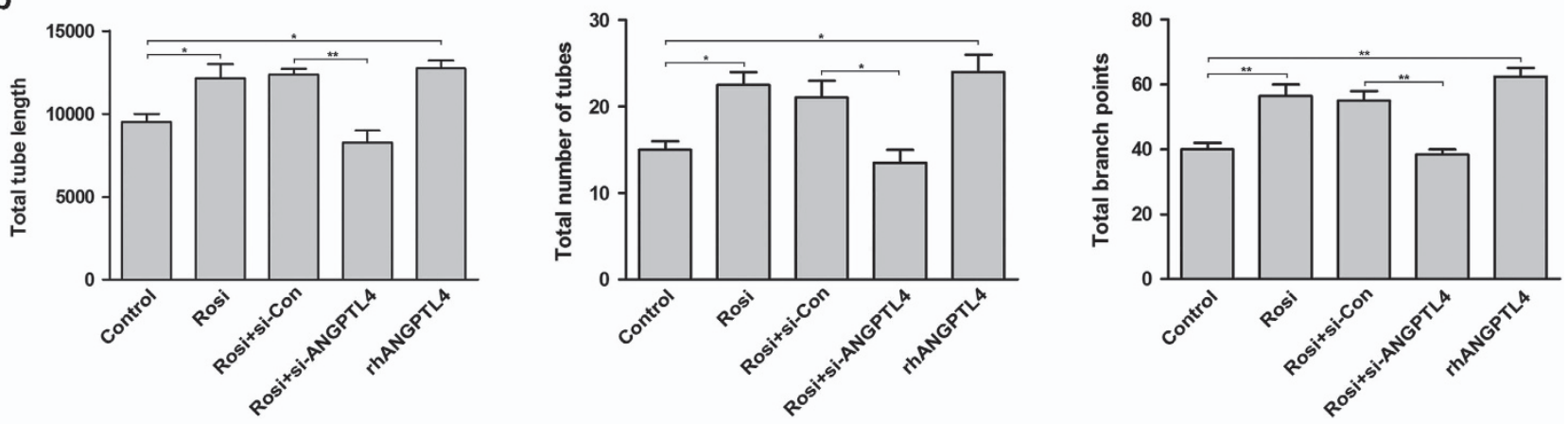

C
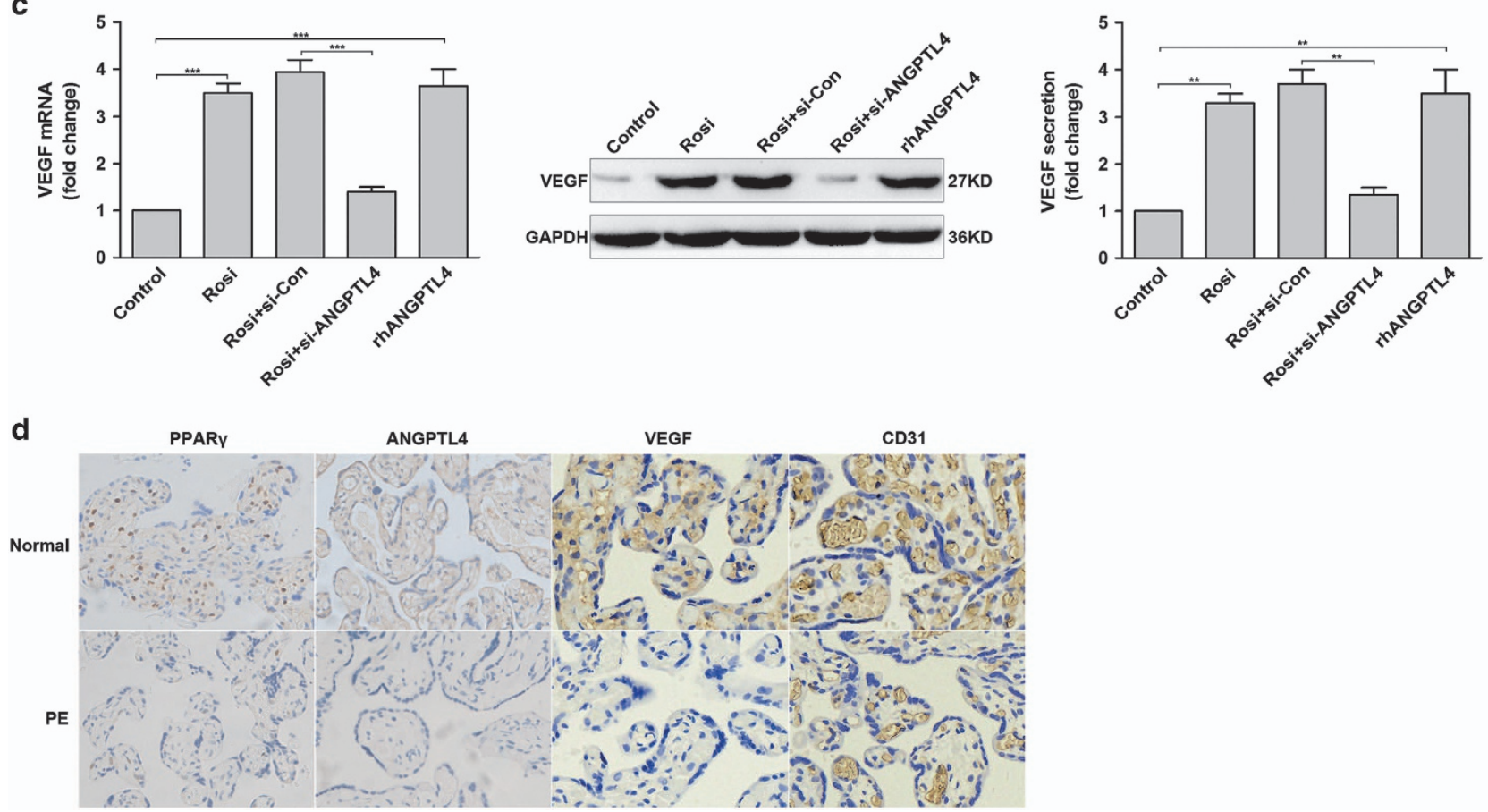

Figure 6 ANGPTL4 plays an important role in rosiglitazone-induced angiogenesis. (a-c) HUVECs transfected with si-Con or si-ANGPTL4 were stimulated with $1 \mu \mathrm{M}$ rosiglitazone. Control cells were treated with $1 \mu \mathrm{M}$ rosiglitazone or $100 \mathrm{nM}$ rhANGPTL4. Tube formation assay was performed in these cells, and then the mRNA and protein levels of VEGF and its secretion were evaluated through western blot, qRT-PCR and ELISA. The data are shown as the means \pm S.E.M. ${ }^{*} P<0.05,{ }^{* *} P<0.01$ and ${ }^{* \star *} P<0.001$ relative to control or si-Con. (d) The expression of PPAR $\gamma$, ANGPTL4, VEGF and CD31 in placental tissues was examined by IHC in PE subjects $(n=30)$ and normal controls $(n=30)$. Representative images were captured at $\times 400$ magnification

is important to note that these results are in good agreement with previous observations. ${ }^{8}$ In addition, decreased PPARy activators in the serum may be responsible for the decrease in PPARy and the expression and secretion of ANGPTL4 in PE.

It is widely accepted that PE is associated with excessive apoptosis and poor invasion of trophoblast cells and limited remodelling of spiral arteries. ${ }^{3}$ Therefore, we sought to explore the functional roles of PPAR $y$ and ANGPTL4 in these aspects. Oxidative stress is responsible for increased trophoblast cell apoptosis. ${ }^{17}$ In the present study, hydrogen peroxide, an oxidative stress marker, was used to treat HTR8/SVneo cells. The results showed that ANGPTL4 mediated the role of rosiglitazone in anti-apoptosis, accompanying with the decreased expression of genes associated with apoptosis 


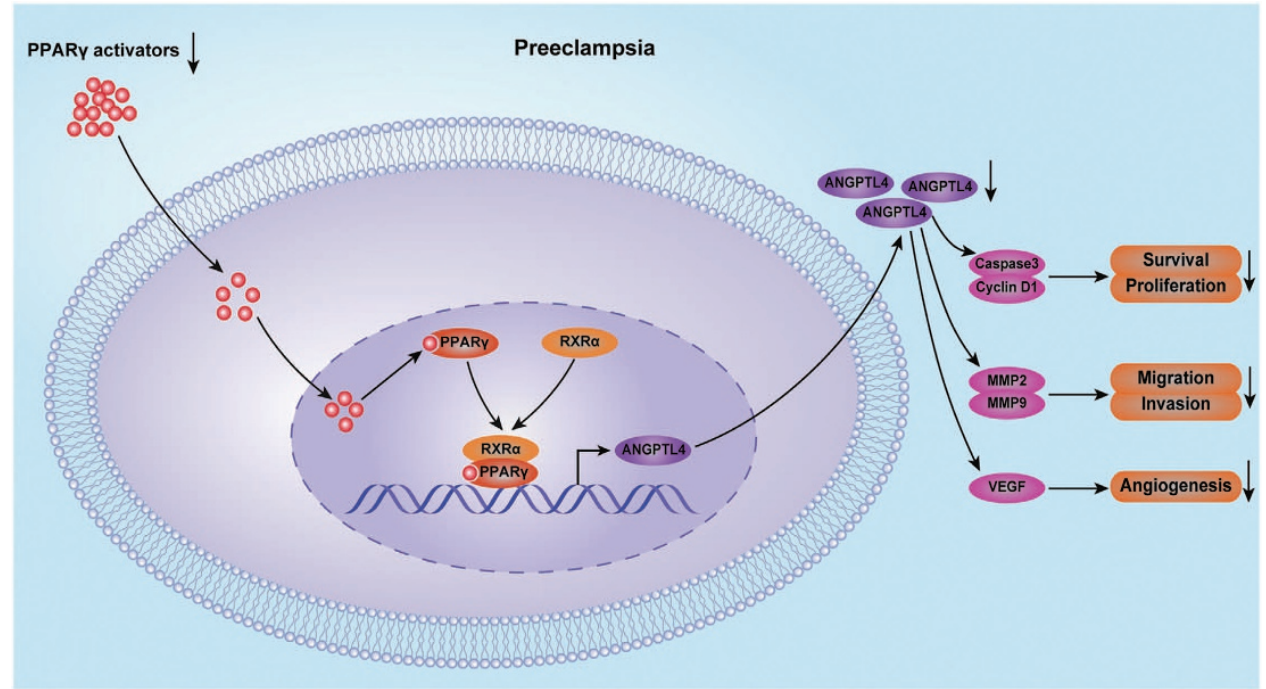

Figure 7 Schematic diagram of ANGPTL4 mediated the protective role of PPAR $\gamma$ activators in the pathogenesis of PE. PPAR $\gamma$ agonist induces ANGPTL4 expression by regulating the binding of the PPAR $\gamma / \mathrm{RXR} \alpha$ heterodimer to its promoter region and enhancing its transcriptional activity. Moreover, our data show that the expression and circulating activators of PPAR $\gamma$ and the expression and secretion of ANGPTL4 are decreased in PE subjects. In addition, we provide evidence that ANGPTL4 mediates the facilitative effects of the PPAR $\gamma$ agonist on regulating the survival, proliferation, migration and invasion of HTR8/SVneo cells, placental explant outgrowth and angiogenesis in HUVECS

(cleaved PARP, caspase-9, cleaved caspase-9, caspase-3, cleaved caspase-3 and Bax). Similar to previous findings, ${ }^{14}$ we found that ANGPTL4 mediated the facilitative effects of rosiglitazone on trophoblast cells proliferation, with increased expression of proliferation-related genes (Cyclin D1, Bcl-2, $\mathrm{c}-\mathrm{Myc}$ and $\mathrm{pHH} 3$ ). Moreover, IHC revealed that preeclamptic placental tissue with low expression of PPARy and ANGPTL4 displayed increased caspase-3 expression and decreased Cyclin D1 expression compared with normal controls.

Given that poor invasion of trophoblast cells and limited remodelling of the spiral arteries are the main pathological features of $P E .^{3}$ For migration and invasion, we found that ANGPTL4 mediated the PPARy agonist-induced the migration and invasion of trophoblast cells and placental explant outgrowth. MMPs are required to create a microenvironment that enables the migration and invasion of trophoblast cells, and decreased MMP-2 and MMP-9 expression in preeclamptic placental tissue has been demonstrated. ${ }^{24,25}$ Analogously, our results suggest that ANGPTL4 mediates PPAR $y$ agonistinduced MMP-2 and MMP-9 expression. Moreover, IHC analysis verified decreased MMP-2 and MMP-9 expression among preeclamptic placental tissues with lower PPARy and ANGPTL4 levels. VEGF serves as a predominant regulator of angiogenesis and plays a crucial role in the production of blood vessels. ${ }^{26}$ Meanwhile, PE is associated with decreased serum level of VEGF and lower microvessel counts in placentas compared with normal controls. ${ }^{27,28}$ Previous studies also provide evidence that ANGPTL4 mediates PPARy agonist-induced angiogenesis. ${ }^{13,18}$ These data are consistent with our present findings in which ANGPTL4 was determined to be involved in PPARy agonist-induced angiogenesis by regulating VEGF expression. In addition, decreased expression of VEGF and CD31 were observed in preeclamptic placental tissues with low PPARy and ANGPTL4 expression.
In summary, we demonstrate that the PPARy agonist induces ANGPTL4 expression by regulating the binding of the PPAR $/ \mathrm{RXR} a$ heterodimer to its promoter region and enhancing its transcriptional activity. Moreover, our data show that the expression and circulating activators of PPAR $y$ and the expression and secretion of ANGPTL4 are decreased in PE subjects. It is worth noting that reduced ANGPTL4 expression and secretion may be attributed to decreased circulating activators of PPARy in PE. In addition, we provide evidence that ANGPTL4 mediates the facilitative effects of the PPARy agonist on regulating the survival, proliferation, migration and invasion of HTR8/SVneo cells, placental explant outgrowth and angiogenesis in HUVECs. Previous study has reported that rosiglitazone administration ameliorated hypertension, improved vascular function and prevented the development of several of the pathophysiological characteristics associated with the rat model of PE. ${ }^{9}$ Therefore, PPARy and ANGPTL4 may become potential novel targets for prevention and treatment of PE. However, further studies are required to investigate if PPAR $y$ agonist is effective in prevention and treatment of women with PE.

\section{Materials and Methods}

Collection of placental tissues and blood samples. A total of 60 placental tissues and corresponding blood samples, including $30 \mathrm{PE}$ and 30 normal controls, were collected at Ren Ji Hospital, School of Medicine, Shanghai Jiao Tong University. All placental tissues and blood samples were collected according to protocols approved by the Research Ethic Committee of the School of Medicine, Shanghai Jiao Tong University. Written informed consent was obtained from all patients. The clinical features of patients were displayed in Supplementary Table 1.

Cell and placental explant culture. HTR8/SVneo (an immortalised human trophoblast cell line) and HUVECs were cultured in DMEM/F12 supplemented with $10 \%$ FBS. Placental explants were cultured as previously described. ${ }^{29,30}$ In brief, tissues from first-trimester placental villi were dissected and explanted onto transwell inserts or a 24-well culture dish pre-coated with Matrigel. Explants were 
permitted to attach to the Matrigel for $4 \mathrm{~h}$ and were then supplied with culture medium without serum. Explants were treated with disparate approaches based on the experimental purpose.

RNA extraction and qRT-PCR. Total cellular RNA was extracted using TRIzol reagent according to the manufacturer's instructions. Primers for PPAR $\gamma$, ANGPTL4, VEGF, Cyclin D1, GAPDH and caspase-3 were obtained from Invitrogen Bioengineering Corporation (Shanghai, China). The sequences of the primers being used for PCR reactions were listed in Supplementary Table 2. qRT-PCR was performed as previously described. ${ }^{31}$

Western blot analysis. Protein preparation and western blot analysis were performed as previously described. ${ }^{31}$ Antibodies against CK7, PPAR $\gamma$, ANGPTL4, RXR $\alpha$, Cyclin D1, Bax, Bcl-2, caspase-3, caspase-9, cleaved caspase-3, cleaved PARP, c-Myc, pHH3, MMP-2, MMP-9, TIMP-1, TIMP-2, $\beta$-actin and GAPDH were obtained from Abcam (Cambridge, UK). Antibodies against VEGF and cleaved caspase-9 were obtained from Millipore (Billerica, MA, USA) and Cell Signalling Technology (Boston, MA, USA), respectively.

ELISA. ELISAs (Abcam) were used to detect the levels of ANGPTL4 and VEGF and were performed according to the manufacturer's instructions. The concentration of each sample was determined by measuring the absorbance at $450 \mathrm{~nm}$ in a microplate reader. Each sample was run in triplicate.

Immunohistochemical and immunofluorescence staining. IHC and immunofluorescence were performed as previously described. ${ }^{32}$ For immunofluorescence analysis, whole mount immunofluorescent staining was performed to confirm the role of PPAR $\gamma$ in ANGPTL4 expression in placental explants. In brief, placental explants cultured for $72 \mathrm{~h}$ in the presence of phenol red-free Matrigel were fixed with $4 \%$ paraformaldehyde. Subsequently, the explants were washed, blocked, and incubated with the primary antibody and fluorescent secondary antibodies. Ultimately, the fluorescent signals were photographed on an inverted microscope.

ChIP. ChIP was carried out as previously described. ${ }^{33}$ Three sets of PCR primers were designed to represent different regions of the ANGPTL4 promoter: PPRE1, PPRE2 and PPRE3. Primer sequences were shown in Supplementary Table 2.

TUNEL and cell proliferation assays. The TUNEL assay was performed according to the manufacturer's instructions (Sigma-Aldrich, St. Louis, MO, USA). Cell proliferation was determined using the Cell Counting Kit-8 (CCK-8) assay (Dojindo, Rockville, MD, USA) according to the manufacturer's instructions.

Transwell invasion and wound-healing assays. Wound healing and transwell invasion assays were executed as previously described. ${ }^{34}$ Cell invasion was detected using a cell culture insert (Corning Incorporated, USA) coated with Matrigel (BD Biosciences, San Jose, CA, USA).

Gelatin zymography assay. Gelatin zymography was carried out as previously reported..$^{32}$ In brief, the supernatants were collected and electrophoresed on SDS-polyacrylamide gels with $1 \%$ gelatin. Subsequently, the gels were washed, incubated, stained and destained successively.

Tube formation assay. The tube formation assay was performed as follows: growth factor reduced Matrigel (BD Biosciences) was placed in a 96-well cell culture plate $\left(60 \mu \mathrm{l} /\right.$ well) and incubated at $37^{\circ} \mathrm{C}$ for $30 \mathrm{~min}$. HUVECs transfected with si-Con or si-ANGPTL4 (10 000/200 $\mu \mathrm{l})$ were seeded onto the Matrigel-coated wells. HUVECs served as a control. Cells were treated with rosiglitazone or recombinant human ANGPTL4 and incubated at $37^{\circ} \mathrm{C}$ for $20 \mathrm{~h}$. Tube formation was observed under an inverted microscope.

Statistical analysis. All data are shown as mean \pm S.E.M. All statistical analyses, including one-way ANOVA, t-test and Pearson's correlation, were executed using SPSS 20.0. All experiments were independently repeated at least in triplicate. Differences between groups were thought to be statistically significant at $P<0.05$.

\section{Conflict of Interest}

The authors declare no conflict of interest.
Acknowledgements. This work was supported by the National Key Technology Research and Development Program of the Ministry of Science and Technology of China during the '12th Five-Year Plan' (2014BAl05B05).

\section{Publisher's Note}

Springer Nature remains neutral with regard to jurisdictional claims in published maps and institutional affiliations.

1. Steegers EA, von Dadelszen P, Duvekot JJ, Pijnenborg R. Pre-eclampsia. Lancet 2010; 376: 631-644

2. He P, Chen Z, Sun Q, Li Y, Gu H, Ni X. Reduced expression of 11beta-hydroxysteroid dehydrogenase type 2 in preeclamptic placentas is associated with decreased PPARgamma but increased PPARalpha expression. Endocrinology 2014; 155: 299-309.

3. Yu L, Li D, Liao QP, Yang HX, Cao B, Fu G et al. High levels of activin A detected in preeclamptic placenta induce trophoblast cell apoptosis by promoting nodal signaling J Clin Endocrinol Metab 2012; 97: E1370-E1379.

4. Mangelsdorf DJ, Thummel C, Beato M, Herrlich P, Schutz G, Umesono K et al. The nuclear receptor superfamily: the second decade. Cell 1995; 83: 835-839.

5. Cipolla MJ, Bishop N, Vinke RS, Godfrey JA. PPAR\{gamma $\}$ activation prevents hypertensive remodeling of cerebral arteries and improves vascular function in female rats. Stroke 2010; 41: 1266-1270.

6. Marx N, Bourcier T, Sukhova GK, Libby P, Plutzky J. PPARgamma activation in human endothelial cells increases plasminogen activator inhibitor type-1 expression: PPARgamma as a potential mediator in vascular disease. Arterioscler Thromb Vasc Biol 1999; 19 . 546-551.

7. Schaiff WT, Carlson MG, Smith SD, Levy R, Nelson DM, Sadovsky Y. Peroxisome proliferator-activated receptor-gamma modulates differentiation of human trophoblast in a ligand-specific manner. J Clin Endocrinol Metab 2000; 85: 3874-3881.

8. Waite LL, Louie RE, Taylor RN. Circulating activators of peroxisome proliferator-activated receptors are reduced in preeclamptic pregnancy. J Clin Endocrinol Metab 2005; 90 : 620-626.

9. McCarthy FP, Drewlo S, Kingdom J, Johns EJ, Walsh SK, Kenny LC. Peroxisome proliferator-activated receptor-gamma as a potential therapeutic target in the treatment of preeclampsia. Hypertension 2011; 58: 280-286.

10. Li H, Ge C, Zhao F, Yan M, Hu C, Jia D et al. Hypoxia-inducible factor 1 alpha-activated angiopoietin-like protein 4 contributes to tumor metastasis via vascular cell adhesion molecule-1/integrin beta1 signaling in human hepatocellular carcinoma. Hepatology 2011; 54: $910-919$.

11. Hato $\mathrm{T}$, Tabata $\mathrm{M}$, Oike $\mathrm{Y}$. The role of angiopoietin-like proteins in angiogenesis and metabolism. Trends Cardiovasc Med 2008: 18: 6-14.

12. Goh YY, Pal M, Chong HC, Zhu P, Tan MJ, Punugu L et al. Angiopoietin-like 4 interacts with matrix proteins to modulate wound healing. J Biol Chem 2010; 285: 32999-33009.

13. Tian L, Zhou J, Casimiro MC, Liang B, Ojeifo JO, Wang M et al. Activating peroxisome proliferator-activated receptor gamma mutant promotes tumor growth in vivo by enhancing angiogenesis. Cancer Res 2009; 69: 9236-9244.

14. Ben-Zvi D, Barrandon O, Hadley S, Blum B, Peterson QP, Melton DA. Angpt/4 links alphacell proliferation following glucagon receptor inhibition with adipose tissue triglyceride metabolism. Proc Natl Acad Sci USA 2015; 112: 15498-15503.

15. Yamazaki K, Shimizu M, Okuno M, Matsushima-Nishiwaki R, Kanemura N, Araki $\mathrm{H}$ et al. Synergistic effects of RXR alpha and PPAR gamma ligands to inhibit growth in human colon cancer cells-phosphorylated RXR alpha is a critical target for colon cancer management Gut 2007; 56: 1557-1563.

16. Schaiff WT, Bildirici I, Cheong M, Chern PL, Nelson DM, Sadovsky Y. Peroxisome proliferator-activated receptor-gamma and retinoid $X$ receptor signaling regulate fatty acid uptake by primary human placental trophoblasts. J Clin Endocrinol Metab 2005; 90 : 4267-4275.

17. Melland-Smith M, Ermini L, Chauvin S, Craig-Barnes $H$, Tagliaferro A, Todros $T$ et al. Disruption of sphingolipid metabolism augments ceramide-induced autophagy in preeclampsia. Autophagy 2015; 11: 653-669.

18. Gealekman O, Burkart A, Chouinard M, Nicoloro SM, Straubhaar J, Corvera S. Enhanced angiogenesis in obesity and in response to PPARgamma activators through adipocyte VEGF and ANGPTL4 production. Am J Physiol Endocrinol Metab 2008; 295: E1056-E1064.

19. Limor R, Sharon O, Knoll E, Many A, Weisinger G, Stern N. Lipoxygenase-derived metabolites are regulators of peroxisome proliferator-activated receptor gamma-2 expression in human vascular smooth muscle cells. Am J Hypertens 2008; 21: 219-223.

20. Frenzel E, Wrenger S, Immenschuh S, Koczulla R, Mahadeva R, Deeg HJ et al. Acute-phase protein alpha1-antitrypsin-a novel regulator of angiopoietin-like protein 4 transcription and secretion. J Immunol 2014; 192: 5354-5362.

21. Barak Y, Nelson MC, Ong ES, Jones YZ, Ruiz-Lozano P, Chien KR et al. PPAR gamma is required for placental, cardiac, and adipose tissue development. Mol Cell 1999; 4: 585-595.

22. Tarrade A, Schoonjans K, Pavan L, Auwerx J, Rochette-Egly C, Evain-Brion D et al. PPARgamma/RXRalpha heterodimers control human trophoblast invasion. J Clin Endocrinol Metab 2001; 86: 5017-5024. 
23. Waite LL, Person EC, Zhou Y, Lim KH, Scanlan TS, Taylor RN. Placental peroxisome proliferator-activated receptor-gamma is up-regulated by pregnancy serum. J Clin Endocrinol Metab 2000; 85: 3808-3814.

24. Tian FJ, Qin CM, Li XC, Wu F, Liu XR, Xu WM et al. Decreased stathmin-1 expression inhibits trophoblast proliferation and invasion and is associated with recurrent miscarriage. Am J Pathol 2015; 185: 2709-2721.

25. Yang Y, Zhang J, Gong Y, Liu X, Bai Y, Xu W et al. Increased expression of prostasin contributes to early-onset severe preeclampsia through inhibiting trophoblast invasion. $J$ Perinatol 2015; 35: 16-22.

26. Ferrara N. Vascular endothelial growth factor as a target for anticancer therapy. Oncologist 2004; 9: 2-10.

27. Torry DS, Wang HS, Wang TH, Caudle MR, Torry RJ. Preeclampsia is associated with reduced serum levels of placenta growth factor. Am J Obstet Gynecol 1998; 179: 1539-1544.

28. Uras N, Oguz SS, Zergeroglu S, Akdag A, Polat B, Dizdar EA et al. CD31 and Factor VIII in angiogenesis of normal and pre-eclamptic human placentas. J Obstet Gynaecol 2012; 32 533-536.

29. Bilban M, Haslinger P, Prast J, Klinglmuller F, Woelfel T, Haider S et al. Identification of novel trophoblast invasion-related genes: heme oxygenase-1 controls motility via peroxisome proliferator-activated receptor gamma. Endocrinology 2009; 150: 1000-1013.

30. Nicola C, Timoshenko AV, Dixon SJ, Lala PK, Chakraborty C. EP1 receptor-mediated migration of the first trimester human extravillous trophoblast: the role of intracellular calcium and calpain. J Clin Endocrinol Metab 2005; 90: 4736-4746.

31. Liu L, Zhang J, Fang C, Zhang Z, Feng Y, Xi X. OCT4 mediates FSH-induced epithelialmesenchymal transition and invasion through the ERK1/2 signaling pathway in epithelia ovarian cancer. Biochem Biophys Res Commun 2015; 461: 525-532.
32. Liu S, Wang J, Qin HM, Yan XM, Yang XS, Liu C et al. LIF upregulates poFUT1 expression and promotes trophoblast cell migration and invasion at the fetal-maternal interface. Cell Death Dis 2014; 5: e1396.

33. Liu NA, Araki T, Cuevas-Ramos D, Hong J, Ben-Shlomo A, Tone Y et al. Cyclin E-mediated human proopiomelanocortin regulation as a therapeutic target for cushing disease. J Clin Endocrinol Metab 2015; 100: 2557-2564.

34. Luo L, Ye G, Nadeem L, Fu G, Yang BB, Honarparvar E et al. MicroRNA-378a-5p promotes trophoblast cell survival, migration and invasion by targeting Nodal. J Cell Sci 2012; 125: 3124-3132.

c) (i) Cell Death and Disease is an open-access journal published by Nature Publishing Group. This work is licensed under a Creative Commons Attribution 4.0 International License. The images or other third party material in this article are included in the article's Creative Commons license, unless indicated otherwise in the credit line; if the material is not included under the Creative Commons license, users will need to obtain permission from the license holder to reproduce the material. To view a copy of this license, visit http://creativecommons.org/licenses/by/4.0/

(C) The Author(s) 2017

Supplementary Information accompanies this paper on Cell Death and Disease website (http://www.nature.com/cddis) 